\title{
Alles vergeblich!? Religionsdidaktische Konkretionen einer politischen religiösen Bildung für nachhaltige Entwicklung
}

\section{Claudia Gärtner}

Technische Universität Dortmund

Kontakt: Claudia.Gaertner@tu-dortmund.de

eingereicht: 23.03.2021; überarbeitet: 17.06.2021; angenommen: 08.07.2021

\begin{abstract}
Zusammenfassung: Der Einsatz für Klimaschutz und Nachhaltigkeit erscheint oftmals vergeblich, da der individuelle Beitrag angesichts der globalen Herausforderung verschwindend gering ist. (Religiöse) Bildung für nachhaltige Entwicklung, die primär auf Befähigung und Motivation zu individuellem nachhaltigen Handeln zielt, läuft Gefahr, die strukturellen Dimensionen von (Nicht-)Nachhaltigkeit auszublenden, so dass individuelles Handeln eher einen beruhigenden Entlastungseffekt besitzt, ohne nachhaltige, strukturelle Änderungen zu bewirken. (Religiöse) Bildung für nachhaltige Entwicklung ist daher stets auch politisch auszurichten. Theoriegeleitet werden im folgenden Beitrag anhand eines (biografischen, historischen) Fallbeispiels Überlegungen zum religionsdidaktischen Umgang mit Vergeblichkeitserfahrungen bei nachhaltigem Handeln entworfen. Die Reich-Gottes-Botschaft öffnet hierbei einen zentralen hermeneutischen und zugleich utopischen Horizont, der Hoffnung auf eine sozial-ökologische Transformation der Gesellschaft schenkt. Anhand des praktisch-theologischen Dreischritts Sehen - Urteilen - Handeln wird somit die Reich-Gottes-Botschaft anhand des Fallbeispiels religionsdidaktisch transformiert.

Schlagwörter: Bildung für nachhaltige Entwicklung, Ökologisches Lernen, Politisches Lernen, politische Religionspädagogik
\end{abstract}

Abstract: Advocacy for climate protection and sustainability often seems futile, as the individual contribution is miniscule in the face of global challenges. (Religious) education for sustainable development, which primarily aims at enabling and motivating individual sustainable action, runs the risk of ignoring the structural dimensions of (non-)sustainability, so that individual action tends to have a calming relief effect without producing sustainable, structural changes. (Religious) education for sustainable development must therefore always be politically oriented. Guided by theory, the following contribution uses a (biographical, historical) case study to outline considerations on how to deal with experiences of futility in sustainable action within the didactics of religion. The message of the Kingdom of God creates a central hermeneutic and at the same time utopian prospect, which gives hope for a social-ecological transformation of society. By means of the practical-theological three-step approach, i.e. seeing assessing - taking action, the message of the Kingdom of God is thus transformed didactically in terms of religion on the basis of the case study.

Keywords: Education for sustainable development, ecological learning, political learning, political religious pedagogy

„Da muss man auch mit etwas klarkommen, das wir den ,Vergeblichkeitsfaktor' nennen: dass man jeden Tag gegen Windmühlen anrennt. Du kannst die Welt vielleicht ein Stückchen besser machen, aber du kriegst sie nie heil“ (Fuchs \& Hommerich, 2020). So beschreibt ein Polizist seine alltäglichen Erfahrungen. Vergeblichkeit ist auch für die meisten Menschen, die sich für Nachhaltigkeit und eine sozialökologische Transformation der Gesellschaft einsetzen, eine nahezu alltägliche Erfahrung: Ist es nicht vergeblich, im teuren Unverpacktladen einzukaufen, wenn man ungefähr 200 Jahre auf Plastiktüten verzichten muss, um so viel CO2 einzusparen, wie ein Flug nach Mallorca freisetzt (Deeg, 2020)? War die mit 1,4 Millionen Teilnehmer*innen größte Demonstration aller Zeiten im September 2019 in 
Deutschland nicht umsonst, wenn kurz darauf die Bundesregierung ein sog. Klimapaket verabschiedet, mit dem die Ziele des Pariser Klimavertrags niemals eingehalten werden können? Hat selbst radikaler Protest, wie die „Besetzung“ des Dannenröder Forst, eine Chance, die automobilzentrierte Verkehrspolitik zu ändern?

Der zitierte „Vergeblichkeitsfaktor" besitzt in Bildungsprozessen eine hohe Relevanz. Denn auch Schüler*innen erleben, dass ihr Handeln und ihr Engagement oftmals vergeblich ist. Im Folgenden gehe ich der Frage nach, wie diese Vergeblichkeitserfahrungen strukturell-politisch reflektiert und nicht ausschließlich auf individuelles Handeln und entsprechende Motivations- oder Kompensationsmaßnahmen fokussiert werden können. Im ersten Kapitel werden daher diese Vergeblichkeitserfahrungen in einem größeren sozio-kulturellen, ökonomischen Horizont reflektiert, um diese in einem zweiten Kapitel mit einem sozial-ökologischen Zugang zur Reich-Gottes-Botschaft zu verschränken. Hieraus werden abschließend anhand eines konkreten Praxisbeispiels religionsdidaktische Konsequenzen für eine religiöse Bildung für nachhaltige Entwicklung (BNE) gezogen.

\section{Vergeblichkeit nachhaltigen Handelns - oder nachhaltige Nicht-Nachhaltigkeit}

Oftmals wurde bereits die Schwierigkeit beschrieben, dass Menschen nur schwer zum nachhaltigen Handeln zu bewegen sind, da die konkreten Folgen (nicht-)nachhaltigen Handelns individuell kurzfristig kaum spürbar und bislang weitgehend im Globalen Süden wahrnehmbar sind. Zugleich erscheint die Wirkung des eigenen Handelns äußerst gering zu sein (Gärtner, 2020; Kruse-Graumann, 2003). Dennoch zielen viele Ansätze von Bildung für nachhaltige Entwicklung auf die Ebene individuellen Handelns. Ideland \& Malmberg (2015) haben mit "eco-certified child“ analysiert, dass viele Lernsettings Heranwachsende zum vorbildlich handelnden Menschen erziehen möchten. Sie sollen für nachhaltigen Konsum sensibilisiert und motiviert werden, nachhaltiges Handeln wird durchaus als moralisch gut und erstrebenswert konnotiert. Ein Blick in religionspädagogische Materialien unterstreicht dies: Wasser sparen, Second Hand oder ökologisch zertifizierte Kleidung kaufen, Müll sammeln oder fleischarme Ernährung sind Handlungsanregungen, die zumeist affirmativ in Themeneinheiten zur Schöpfungsbewahrung diskutiert werden. Eine so ausgerichtete (religiöse) BNE „trägt zwar zur Beruhigung und zeitweisen Beschäftigung von Kindern bei, verschleiert aber, dass die Probleme größer sind und stärkt damit insgesamt ein Beruhigungsnarrativ“ (Reuter, 2020, S. 322). Folglich erscheinen angesichts der gravierenden planetaren Grenzüberschreitungen, der vielfältigen Umweltkatastrophen und der kaum noch abzuwendenden Klimakatastrophe diese Handlungsanregungen wirklich vergeblich zu sein - erst recht, wenn man berücksichtigt, dass nachhaltiges Handeln längst nicht für alle Schüler*innen erstrebenswert ist. Zwar suggeriert die Bezeichnung "Generation Greta“ (Hurrelmann \& Albrecht, 2020), dass Nachhaltigkeit ein durchgängiges Anliegen heutiger Schüler*innen sei. Demnach sehen ca. 75\% der Jugendlichen Umweltverschmutzung als größtes Problem an und 65\% haben Angst vor dem Klimawandel (Albert, Hurrelmann \& Quenzel, 2019, S. 56). Aber nur ca. 32\% lassen sich als dezidiert nachhaltigkeitsaffine Jugendliche bezeichnen (Michelsen, Grunenberg \& Mader, 2015, S. 3), wobei sie damit nicht zwangsläufig auch einen kleinen ökologischen Fußabdruck besitzen.

Doch ist es nicht besser, zumindest einen (wachsenden) Teil der Schüler*innen zu nachhaltig Handelnden zu erziehen - oder sich Gedanken darüber zu machen, wie die didaktischen Settings verbessert und noch mehr Heranwachsende von Nachhaltigkeit überzeugt werden können? Mit „ecocertified child" werden die Grenzen und Gefahren einer solchen Fokussierung aufgezeigt, da der BNE-Diskurs oftmals einer neoliberalen Rationalität unterliege. „The individual becomes responsible for 'everybody's' security and for the ecological system of the world. This individual focus tends to make conflicts of interests or ideological standpoints invisible“ (Ideland \& Malmberg, 2015, S. 181). BNE, die primär die Verantwortung für Nachhaltigkeit bei den (jungen) Individuen fokussiere, laufe somit nicht nur Gefahr, die Individuen zu überfordern und ggf. moralisch zu überlasten, sondern hiermit könne auch eine 
Entpolitisierung von Nachhaltigkeit einhergehen. „The political muscles are atrophied when the problems are placed on the shoulders of individual children who, despite their lack of income, suffrage, and established channels for making their voices heard in society, are expected to be able to change the world through rational, individual actions. This means that the possibilities for real change may perhaps disappear" (Ideland, 2019, S. 136). Eine so ausgerichtete BNE kann dann zur Stabilisierung gesellschaftlicher Verhältnisse beitragen, die mit dem Konzept der ",nachhaltigen Nicht-Nachhaltigkeit“ (Blühdorn, Buttzlaff, Deflorian, Hausknost \& Mock, 2019) beschrieben werden.

Nach Blühdorn et al. führen derzeit der hegemoniale Kapitalismus bzw. Neoliberalismus dazu, dass ein nachhaltiges Leben in einer nachhaltigen Gesellschaft unterbunden wird. Nicht-Nachhaltigkeit ist vielmehr strukturell nachhaltig abgesichert, wodurch es weder für Individuen noch für Institutionen oder Gesellschaften möglich sei, sich umfassender der kapitalistischen und neoliberalen Wachstumslogik zu entziehen. In dieser Perspektive werden alle auf Konsum eingeschworen, da nur dieser weiteres Wachstum ermögliche, an dem zugleich der erreichte Wohlstand gebunden sei. Der ausgelobte europäische Green New Deal bleibt ebenso in dieser Logik wie eine BNE, die ihren Fokus (primär) auf individuelles nachhaltiges Handeln legt.

Eng hiermit verbunden ist ein zweiter Begründungsansatz für nachhaltige Nicht-Nachhaltigkeit, der als Emanzipation zweiter Ordnung bezeichnet wird. Demnach herrsche in der ökologischen Bewegung seit Beginn ein auf Mündigkeit und Emanzipation gründendes Subjektverständnis vor, das sich für eine sozial-ökologische Gesellschaft stark mache (Blühdorn, 2019). Allerdings scheinen sich bislang die Subjekte weitgehend weder entfremdet zu fühlen noch vom Kapitalismus emanzipiert werden zu wollen. Zumindest fand in den letzten Jahrzehnten eine Befreiung aus Konsumzwängen und kapitalistischen Verquickungen hin zu einem nachhaltigen Leben weitgehend nicht statt. Im Gegenteil: Mit fortschreitender Individualisierung muss sich das Subjekt - ganz emanzipiert - stets neu entwerfen, mit allen Freiheiten und Risiken. Individualisierung, Selbst-Kultur, Risikogesellschaft und Patchworkbiografien sind Schlagwörter, die diese Entwicklung beschreiben. Das unternehmerische Selbst (Bröckling, 2016) muss ständig in sich selbst und seine Singularität investieren, was hohe materielle als auch immaterielle Ressourcen verschlingt und in Spannung zu einer sozial-ökologischen, nachhaltigen Gesellschaft stehen kann. Eng mit dieser Individualisierung verbunden scheint sich das Subjekt auch zunehmend von Verantwortung, Verpflichtungen, Beschränkungen und Prinzipien zu emanzipieren. Es vernachlässigt damit zugleich den aufklärerischen Emanzipationsgedanken, wonach Freiheit und Verantwortung, Mündigkeit und Kritik stets aufeinander bezogen sind. Besonders augenfällig ist dies derzeit, wenn individuelle Freiheiten zum Schutz der Allgemeinheit reguliert werden sollen (Maskenpflicht, Tempolimit u. v. m.). Diese Emanzipation zweiter Ordnung hat Auswirkungen auf die Gestaltung einer (nachhaltigen) demokratischen Gesellschaft, denn hiermit gehen der Demokratie zugleich Voraussetzungen verloren, die sie - entsprechend dem berühmten Böckenförde-Theorem selbst nicht hervorbringen kann (Böckenförde, 1991). Es trägt somit zu einer Demokratiekrise bei, wenn das Wohl des Einzelnen über das Wohl der Gemeinschaft gestellt und Demokratie zunehmend ein Instrument wird, um primär das individuelle Wohlergehen abzusichern.

Hier kann nur in groben Zügen angerissen werden, dass die Klimakrise somit Teil einer multiplen Krise (Demirović, 2013) und eng mit vielfältigen sozialen, ökonomischen, politischen und ökologischen Herausforderungen verwoben ist. (Religiöse) BNE muss diese Komplexität und Vielschichtigkeit von Nachhaltigkeit im Blick behalten, gerade auch wenn ökonomische Ziele in Spannung zu sozialen und ökologischen Nachhaltigkeitszielen stehen. Es ist aufschlussreich, dass in dem neuesten UNESCO-Rahmenpapier zur BNE ungewohnt deutlich die konfliktträchtigen Aspekte der Nachhaltigkeitsziele benannt werden, insbesondere zwischen individuellem nachhaltigen Handeln und nicht-nachhaltigen Strukturen. „As much as attention is required for what is happening at the individual level in relation to transformative decisions and experience, there is a need for ESD to focus more on deep structural causes. 
The relationship between economic growth and sustainable development is one of the pertinent issues in this regard" (UNESCO, 2020, Anex II, Art. 4,8f.). Hier wird zumindest in Ansätzen deutlich, dass der Nachhaltigkeitsbegriff Gefahr läuft, als umbrella term Zielkonflikte zu verdecken, weshalb Nachhaltigkeit derzeit vermutlich auf so breite Zustimmung stößt. Denn der Nachhaltigkeitsbegriff entstammt der Forstwirtschaft und unterliegt von seinem Ursprung her einer ökonomischen Logik (Kluwick \& Zemanek, 2019; Pufé, 2017), indem Natur primär als zu erhaltende Ressource für das ökonomische System betrachtet wird (Prescher, 2019). Ein so konnotierter Nachhaltigkeitsbegriff ist ökonomisch daher äußerst anschlussfähig, ohne zwangsläufig sozial-ökologische Zielsetzungen zu implizieren.

Diese hier nur grob gezeichnete Skizze einer deterministisch nahezu geschlossenen, neoliberal strukturierten Gesellschaft plausibilisiert, warum individuelles, nachhaltiges Handeln vergeblich zu sein scheint. Dennoch zielt BNE auf nachhaltiges Handeln und auch die UN fordert, bis 2030 sicherzustellen, "dass alle Lernenden die notwendigen Kenntnisse und Qualifikationen zur Förderung nachhaltiger Entwicklung erwerben, unter anderem durch Bildung für nachhaltige Entwicklung und nachhaltige Lebensweisen“ (Vereinte Nationen, 2015, Art. 4.7). (Religiöse) BNE bewegt sich daher in einem Spannungsfeld, das zwischen strukturellem Determinismus auf der einen und Veränderungshoffnung durch individuelles Handeln auf der anderen Seite aufgespannt werden kann. BNE richtet sich an gesellschaftlich präformierte Heranwachsende und zielt zugleich auf mündige Subjekte (Gärtner, 2020). Wenn jedoch BNE die Lernenden ausschließlich auf der Ebene des individuellen nachhaltigen Handelns adressiert, läuft sie Gefahr, die nichtnachhaltigen Strukturen zu ignorieren und hierdurch zu reproduzieren. Die Erfahrung, dass nachhaltiges Handeln vergeblich ist oder wirkt, ist in diesem Spannungsfeld zu verorten.

(Religions-)pädagogisch lässt sich dieses Spannungsfeld nicht einfach auflösen, vielmehr muss mit ihm in der (religions-)pädagogischen Praxis umgegangen werden. Hierzu ist es unabdingbar, die Wirkmächtigkeit gesellschaftlicher und ökonomischer Systeme zu kennen, um ihnen nicht blind zu erliegen. Die UNESCO fordert für BNE entsprechend "to raise critical and structural questions" (UNESCO, 2020, Annex II, Art. 5.7). Doch bevor diese Überlegungen religionsdidaktisch konkretisiert werden, soll der Vergeblichkeitsfaktor theologisch reflektiert werden.

\section{Vergeblichkeitserfahrungen und die Reich-Gottes-Botschaft}

Theologisch lassen sich die beschriebenen Spannungen und der Vergeblichkeitsfaktor am besten mit anamnetischen und eschatologischen Denkfiguren beschreiben. Demnach schöpft das Christentum zum einen durch die Erinnerung an die Heilstaten Jesu Hoffnung, dass eine veränderte Welt möglich ist. Zum anderen erinnert die Reich-Gottes-Botschaft daran, dass die menschliche Praxis stets eine vorläufige, nicht vollendete Praxis ist, die der Vollendung durch Gott bedarf und dadurch eben nicht vergebens ist, sondern in Gott aufgehoben und eschatologisch vollendet wird. Im Rahmen der Befreiungstheologie ist dieses anamnetische und eschatologische Reich-Gottes-Potenzial breit entfaltet worden (Sobrino, 1995; 2008) und kann in ähnlicher Weise auf sozial-ökologische Krisen bezogen werden (Amlinger, 2017; Moltmann, 1985). Demnach fordern Jesu Handeln und seine Zeichen zur Nachfolge Jesu auf. Christ*innen ist aufgetragen, an der Realisierung des Reiches Gottes mitzuwirken. Jesus vertröstet nicht auf das Jenseits, sondern er setzt sich für Marginalisierte, Kranke, Arme in seinem direkten Umfeld ein. Insbesondere die Wundererzählungen sowie die Gleichnisse besitzen dabei den Anspruch, etwas von der Wirklichkeit des Reiches Gottes zu zeigen. „Aber diese Zeichen sind nicht deckungsgleich mit der Wirklichkeit des Reiches. Weder schaffen die Heilungen die Realität der Krankheit ab, noch läßt die Vertreibung der Dämonen die Allgegenwart des Bösen verschwinden [...]. Die Welt als ganze entspricht, um es vorsichtig zu sagen, noch nicht dem Wunsch Gottes. In weiten Teilen ist sie genau das Gegenteil“" (Sobrino, 1995, S. 156f.). Dieses Verhältnis von schon angebrochenem und noch nicht realisierten Reich Gottes wird in den Kontrast- bzw. Wachstumsgleichnissen deutlich. So veranschaulicht das Gleichnis vom Senfkorn (Mk 4,30-32), wie aus einem sehr kleinen Samen ein üppiger Strauch bzw. 
Baum werden kann - und dies ohne Zutun des Menschen. Die Pointe des Gleichnisses liegt jedoch nicht darin, dass dieses Samenkorn bereits gesät ist und die Menschen geduldig auf das Wachsen warten können, auch erschöpft es sich nicht in dem beeindruckenden Kontrast zwischen klein und groß (Müller, Büttner, Heiligenthal \& Thierfelder, 2002, S. 119; Theißen \& Merz, 2011, S. 299), vielmehr „wird die Aufmerksamkeit der Rezipienten auf dieses Ende [des Gleichnisses; Verf.] hin mitgerissen. Die Gottesherrschaft wird, unvorhersehbar, unableitbar, eintreffen und die Welt verwandeln: Diese Hoffnung atmet etwas vom Geist der Utopie“" (Gäbel, 2007, S. 337). Dabei äußert sich dieser utopische Geist in überraschend fremden Bildern. Jesus wählt nicht tradierte Bilder für die Gottesherrschaft, wie z. B. die Zeder als herrschaftliche Pflanze, sondern er veranschaulicht das Reich Gottes mit einer struppigen Senfstaude, die als Gartenpflanze angebaut wird, aber auch als Wildkraut gedeiht. „Das Senfkorn wirkt [...] in geradezu grotesker Weise disproportional, ja vor dem Hintergrund der biblischen Belege für die Zeder als Bild für Herrschergestalten hat der Vergleich mit Senfkorn und -staude etwas fast Armseliges, dadurch aber auch Ironisch-Subversives, das geeignet ist, Träume von imperialer Größe in ein komisches Licht zu setzen“ (Gäbel, 2007, S. 338).

Die Reich-Gottes-Botschaft Jesu sprengt somit die menschlichen Vorstellungen, ihr ist ein visionär-utopisches Potenzial zu eigen. Indem Jesus durch die selbstwachsende Saat auf das mächtige und erlösende Handeln Gottes verweist, spendet er Hoffnung auf das Reich Gottes, das die menschlichen Vorstellungen übersteigt. Zugleich liegt in dieser Hoffnung und Utopie Kraft und Auftrag für menschliches Handeln. „Nur wer Hoffnung hat, kann in hoffnungslos erscheinenden, verfahrenen Situationen einerseits die Utopien ablegen, die hier nicht mehr gehen, und andererseits sich auf das hin umstellen, was jetzt hier nottut" (Sander, 2016, S. 28).

Anamnetisches und eschatologisches Denken durchbricht somit sowohl alternativloses Denken, wonach es zur gegenwärtigen, z. B. ökonomischen Praxis keine Alternativen gebe, als auch resignatives Denken, wonach individuelles Handeln vergeblich sei. In der Spannung zwischen dem Auftrag zum Handeln für eine gerechtere Welt und dem Versprechen, dieses nicht allein verantworten und vollenden zu müssen, liegt sowohl Kraft und Hoffnung als auch Schutz vor Überforderung, die zu „strukturellen Selbstimmunisierungs-, Selbstentschuldigungs- und Selbstentschuldungsprozesse[n]“ (Fuchs, 1995, S. 163) führen kann, wie dies gerade beim Klimaschutz derzeit auf vielen Ebenen wahrnehmbar ist. Auf Gott und seine Reich-Gottes-Botschaft zu setzen, ist somit „Ausdruck einer kontrafaktischen, widerständigen Hoffnung, die gerade darin zutiefst human ist, dass sie den Menschen vom Zwang zur Selbstund Welterlösung befreit“" (Körtner, 2020).

Eine solche theologische Deutung von und der Umgang mit Vergeblichkeit lässt sich mit dem soziologischen Begriff einer refigurativen Praxis in Beziehung setzen. Im Rahmen der Transformationsforschung wird unter diesem Begriff das „Immer-wieder-bilden“ (Deflorian, 2019, S. 215) einer sozial-ökologischen Lebenspraxis verstanden. Demnach stellen sozial-ökologische Transformationsprojekte eine refigurative Praxis dar, sie sind das „wiederholende Schaffen einer utopischen Gegenwart in einer hochdynamischen Gesellschaft“ (Deflorian, 2019, S. 219). Refigurative Praxis grenzt sich von der Vorstellung einer präfigurativen Praxis ab, wonach diese eine Transformationsgesellschaft präfigurieren könnte (Yates, 2015). In diesem Verständnis werden in transformativen Keimzellen, in Nischenprojekten, alternative Gesellschaftsformen vorher-gebildet, die dann sukzessive die Gesamtgesellschaft verändern können. In den letzten Jahrzehnten konnten jedoch solche Nischenprojekte kaum zur Veränderung der Gesellschaft beitragen, angesichts des hegemonialen Kapitalismus stoßen diese immer wieder an ihre Grenzen. In nahezu befreiungstheologischer Terminologie schließt Deflorian hieraus: „Das heißt nicht, die Hoffnung auf einen solchen Wandel aufzugeben. Sondern es bedeutet, sich von dieser Hoffnung nicht den offenen Blick auf gesellschaftliche Phänomene nehmen zu lassen“ (Deflorian, 2019, S. 222). Der Begriff der refigurativen Praxis greift diese Spannung von notwendigem, hoffnungsvollem Handeln, Gesellschaftsanalyse und Scheitern auf. Religiöse BNE wird daher im Folgenden in dieser Per- 
spektive auch als eine refigurative Praxis entworfen, die sich in eschatologischer Weise der Beteiligung „schon" an der Reich-Gottes-Botschaft verschreibt im Wissen, dass sie diese "noch nicht" umfassend präfigurieren oder realisieren kann.

\section{Religionsdidaktische Konkretionen}

Religionsdidaktisch gilt es, diese skizzierten Spannungen nicht zu umgehen oder in unterkomplexen (religiösen) Handlungsimpulsen aufzulösen. Vielmehr müssen die skizzierte Determiniertheit von Subjekt und Gesellschaft im hegemonialen Kapitalismus sowie die Herausforderungen angesichts der sog. Emanzipation zweiter Ordnung in religiösen Bildungsprozessen mitbedacht und zugleich kritisch transparent gemacht werden, gerade auch wenn diese in Hinblick auf einen religiösen Umgang mit der (vermeintlichen) Vergeblichkeit nachhaltigen Handelns reflektiert werden. Die im Folgenden entfalteten religionsdidaktischen Konkretionen orientieren sich am etablierten praktisch-theologischen Dreischritt Sehen, Urteilen und Handeln. Dabei zielen die initiierten Lernprozesse, die für Schüler*innen ab der Jahrgangsstufe 9 anzusetzen sind, auf eine christlich motivierte, refigurative Praxis, die sowohl die Vergeblichkeitserfahrungen aufgreift als auch kritisch nicht-nachhaltige Strukturen und Zielkonflikte analysiert und im Horizont der anamnetisch-eschatologischen christlichen Botschaft interpretiert.

Im ersten Schritt sollen die Schüler*innen zu einer kritischen Wahrnehmung von Nachhaltigkeit befähigt werden. Es gilt dabei auch, die jeweils interessegeleiteten Perspektiven und Kontexte in den Blick zu nehmen und "die ideologiekritische Einsicht über den Zusammenhang von Erkenntnis und Interesse“ (Boschki, 2008, S. 88) einzuholen. Sehen umfasst somit stets auch die kritische (Selbst-)Reflexion des Wahrgenommenen.

Um Schüler*innen einen kritischen Blick auf Nachhaltigkeit und die hierdurch ggf. verschleierten Zielkonflikte, die zu einer nachhaltigen Nicht-Nachhaltigkeit sowie zu Vergeblichkeitserfahrungen führen können, zu eröffnen, wird in dem Lernsetting eine Kontextverschiebung vorgenommen und ein historisches Fallbeispiel analysiert. Dabei kann das Lernsetting an religionsdidaktisch etablierte Prinzipien anknüpfen, wie z. B. biografisch orientiertes, kirchengeschichtliches Lernen (Lindner, 2007; Bork \& Gärtner, 2016) oder Modelllernen (Mendl, 2015). Insbesondere wenn Fallbeispiele, wie im Folgenden, aus lokalen lebensweltlichen Kontexten oder aus biografischen Schilderungen entstammen, greifen hier zudem lernförderliche Faktoren wie z. B. Emotionalität, Lebensweltbezug, Exemplarität und modellhaftes Lernen, wie sie auch im Rahmen von (religiöser) BNE aufgegriffen und unterstrichen werden (Bederna, 2019; Gärtner, 2020).

Beim Blättern durch meine Familienchronik fielen mir Schilderungen meines Ururgroßvaters (Abb.1) vom Leben in einem kleinen Sauerländer Dorf im 19. Jahrhundert auf, die im Folgenden zu einem Fallbeispiel religiöser BNE verdichtet werden sollen. Mit großer Missbilligung beschreibt mein Vorfahre, ein einfacher Bauer, wie in nur wenigen Jahren große Teile des Mischwaldes abgeholzt wurden, um hiermit die Hochöfen im Siegerland zu heizen. Nachdem große Teile des Waldes aus kommunaler Hand in Privatbesitz übergeführt wurden, „war in den 1850er Jahren eine verdienstreiche Zeit. In den Wäldern war viel Arbeit und nach damaliger Zeit wurde viel verdient. [...] Zugleich hatte in den 50er Jahren der Feind, die Axt, den Sieg davon getragen und tausende lebenskräftige Naturen zu Boden geschmettert, und die sonst so schönen Standorte waren öde kahle Bergrücken, die mit wildem Gestrüpp bewachsen zurück gelassen [wurden]. Da nun im Jahre 1860-65

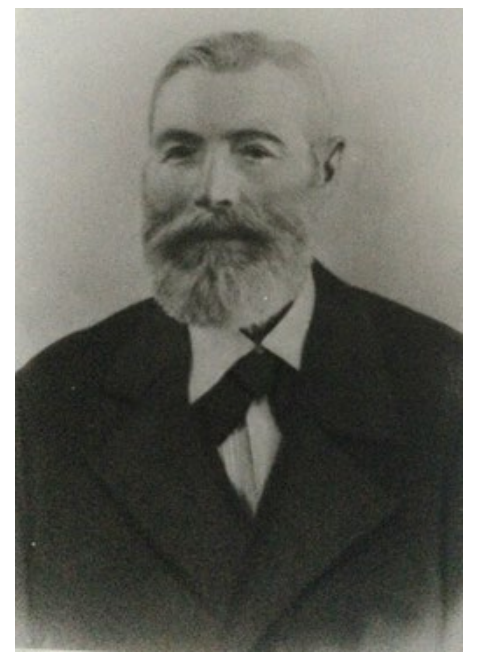

Abb. 1: Georg Rüther (1834-1920) das Gesuchte Holz war, weil es zu Bauten und Holzschleifereien und sonst gewerblichen Zwecken genutzt wurde, wurden die öden Stellen mit Tann bepflanzt. So sieht man nun überall Tannenbestände. 
[...] Seitdem die Waldblößen mit Tannen angepflanzt wurden, ist die Bienenzucht nicht mehr so ertragreich.“ Auch die „Massentierhaltung“ beklagt er, da die kargen landwirtschaftlichen Erträge die Tiere, die Rede ist von 4 Pferden und 15-20 Stück Rindvieh, im Winter nicht ernähren könnten und deshalb oftmals das Vieh jämmerlich stürbe. Doch im „Jahre 1849 trat ein Wendepunkt ein. Ein vernünftig denkender Landwirt sah ein, wo der Hase im Pfeffer lag, daß so wenig von der Landwirtschaft erzielt wurde, daß nur die verkehrte Viehzucht dieses Übel zu Wege brachte." Mein Ururgroßvater macht einen gewichtigen Grund für die aufgezeigten Missstände aus: „Der gute Verdienst, die gute Löhnung, die jetzt überall herrscht, ist Schuld an dem obigen Übel. Jetzt sieht man bei den Mädchen, wenn sie heiraten (mit 18-20 Jahren wollen sie schon einen Mann haben) keine Koffer voll wertvolles Leinen und wertvolle Kleider, sondern stattdessen eine Anzahl Modehüte und Modekleider. Mit allem Modernen und Luxuriösen muß das sein."

Erschreckend aktuell erscheint die Chronik: Waldrodung, Monokultur, Verlust der Biodiversität, Massentierhaltung, kurzfristige ökonomische Interessen, Privatisierung von Gemeingut, Konsumstreben ... Obwohl die Missstände bereits seit über 150 Jahren bekannt sind, sind diese Praktiken und Strukturen immer noch wirkmächtig, mehr noch: Die Ausmaße der ökologischen Krise und ihre ökonomischen und sozialen Verstrickungen sind gewaltiger und katastrophaler geworden. Wäre allein hieran schon der Vergeblichkeitsfaktor diskutierbar, so wird dieser angesichts des hoffnungsvollen Ausblicks meines Vorfahrens noch gesteigert. „Wie es in der vergangenen Zeit in Allem fortgeschritten ist, so wird in ferner Zeit auch die Wissenschaft noch vollkommener werden und neue Erfindungen und neue Werke schaffen. So wird man sich auch dann von dieser Zeit, 1910 sagen, wie wir jetzt sprechen von der Zeit 1830. Jetzt ist doch die Zeit viel, viel aufgeklärter als damals. Große Reiche haben einen Rückschlag erhalten und sind sogar zu nichts geworden. Aber die Wissenschaft hat nie einen Stillstand erhalten, sondern ist von je her vorangeschritten und wird weiter voran schreiten bis zum Ende der Zeit." Doch auch die Hoffnung auf wissenschaftlichen und technologischen Fortschritt wurde - zumindest in Hinblick auf die ökologische Frage - nicht eingelöst. Durch den sog. Rebound-Effekt führen z. B. neue Technologien zu Energieeffizienz, jedoch brauchen vermehrter Konsum oder veränderte Nutzungsgewohnheiten diese Energieersparnis weitgehend auf. Nachhaltiges Wirtschaftswachstum durch technologischen Fortschritt, der Wachstum von Ressourcenverbrauch entkoppelt, ist bislang eine Illusion geblieben (Raworth, 2018, S. 293-342).

Wie bereits angedeutet, wurde der Nachhaltigkeitsbegriff in der Forstwirtschaft im 18. Jahrhundert von Hans Carl von Carlowitz geprägt. Hiernach zielt nachhaltige Forstwirtschaft darauf, nur so viele Bäume zu roden, wie nachwachsen können, um auch langfristig hohe Erträge zu sichern. Die historischen Wurzeln und die entsprechende ökonomische Logik des Nachhaltigkeitsbegriffs können die Schüler*innen anhand des beschriebenen oder ähnlicher Fallbeispiele kritisch analysieren und diskutieren (z. B. ökonomischer Fokus, Natur als reine Ressource, Verlust an Biodiversität trotz Aufforstung, Privatisierung von Gemeingut). Sie erarbeiten anhand der historischen Schilderungen, welche Gründe es für (nicht-) nachhaltiges Handeln gab und diskutieren (ggf. fächerübergreifend und mit Zusatzmaterial, um anachronistische Urteile zu vermeiden), inwiefern diese heute auch noch (verändert) zutreffen. Zugleich können sie individuelle Handlungsspielräume erkennen (z. B. verringerte Viehzucht), die zwar punktuell die Situation der Landwirte verbessert, aber dennoch langfristig nicht zu einer nachhaltigeren Lebensweise beigetragen haben. Die Schüler*innen erarbeiten, inwiefern der ökologischen Krise in den letzten 100 Jahren mit technologischem Fortschritt begegnet wurde und welche Auswirkungen wissenschaftliche und technologische Fortschritte auf die Ökobilanz haben (z. B. Emprechtinger \& Papadopoulos, 2020). Ziel ist es in dem ersten Schritt des Sehens, den Nachhaltigkeitsbegriff in seiner Komplexität ideologiekritisch wahrzunehmen, hierin auch Gründe für nachhaltige Nicht-Nachhaltigkeit und Vergeblichkeitserfahrungen zu finden und begrenzte Chancen individuellen nachhaltigen Handelns auszuloten. Wissend, dass ein historischer Vergleich auch zu anachronistischen Urteilen verleiten kann, wird somit durch die historische Kontextverschiebung ein geschärfter Blick auf die Gegenwart und den 
Nachhaltigkeitsdiskurs angezielt.

In einem zweiten Schritt wird der Lernprozess auf die Stufe des (theologischen) Deutens und Urteilens geführt. Dafür lernen die Schüler*innen (Nicht-)Nachhaltigkeit im Horizont der Reich-Gottes-Botschaft zu reflektieren (vgl. Kap. 2). Zwar ist die biblische Rede von der basileia tou theou für Kinder und Jugendliche nicht leicht begrifflich zu erschließen, da Übersetzungen wie „Reich Gottes“, „Königreich“ oder „Himmelreich" metaphorisch nur schwer zugänglich sind und problematische Assoziationen (Dritte Reich, Absolutismus ...; vgl. Lachmann, 2016, S. 298f.; Büttner, 2012) eröffnen. Doch über konkrete Gleichnisse, wie das bereits erwähnten Wachstums- bzw. Kontrastgleichnis (Senfkorn Mk 4,30-32) können Schüler*innen Aspekte der Reich-Gottes-Botschaft konkret erarbeiten. Dabei knüpfen die Naturmetaphern dieses Gleichnisses an dem historischen Fallbeispiel an (Bäume, Wachstum ...). Doch zugleich lässt sich dieses und vergleichbare Gleichnisse im Horizont von Jesu Reich-Gottes-Botschaft nicht präsentisch und allein auf der Bildebene deuten, wonach das Reich Gottes bereits angebrochen und nun dem organischen Wachsen einer Pflanze gleich - auf Erden wächst und gedeiht (Müller et al., 2002, S. 122f.). Vielmehr ist - wie aufgezeigt - die Reich-Gottes-Botschaft immer auch futurisch, steht unter einem eschatologischen Vorbehalt und besitzt einen utopischen Gehalt, der nicht in irdischen Vorstellungen nach Macht und Herrschaft aufgeht. Nicht edle Zedern, wie in der Bibel, oder Tannen, wie im historischen Fallbeispiel, sondern die wildwüchsige Senfstaude wird zum Bild des Reich Gottes. Somit lässt sich die in der Reich-Gottes-Botschaft zum Ausdruck kommende, oftmals kontrafaktische Hoffnung mit ihrem Fremdheits- und Widerstandspotenzial und ihrer Option für die Armen und Ausgegrenzten thematisieren (Lk 14,12-24). „Gerade weil Reich Gottes durch Spannungsverhältnisse gekennzeichnet ist (diesseits und jenseits, schon jetzt und noch nicht, durch Gott herbeigeführt und durch Mensch) und Visionen für die Zukunft bietet, lohnt sich das Unterrichtsgespräch“ (Weiße, 2019, S. 350f.). Die eschatologische Spannung von "schon“ und „noch nicht" realisiertem Reich Gottes wird zur Deutungsfolie, die sowohl zum widerständigen Handeln ermutigt als auch vor Überforderung schützt. In diesem Horizont können Schüler*innen auch ihre Vergeblichkeitserfahrungen (neu) kritisch deuten und beurteilen. Damit wird eine christlich motivierte refigurative Praxis angebahnt, die im dritten Schritt des Handelns vertieft wird.

Um den theologisch höchst anspruchsvollen zweiten Schritt des Urteilens zu konkretisieren und zu exemplifizieren, setzen sich die Schüler*innen im dritten Schritt mit ermutigenden Beispielen christlicher nachhaltiger Praxis auseinander. Damit greift das Lernsetting auf Erkenntnisse der kritischen politischen Bildung sowie BNE zurück, wonach ermutigende und hoffnungsstiftende Lernumgebungen (Besand, 2019) motivierender sind als eine auf Angst zielende „Katastrophendidaktik“ (Gagel, 1994; 2005, S. 123, S. 304). Es überrascht daher nicht, dass auch auf den Bestsellerlisten derzeit Publikationen zu finden sind, die zeigen, wie eine transformierte Gesellschaft umgesetzt werden kann, die ein gutes Leben für alle ermöglicht (Göpel, 2020; Kemfert, 2020). Ermutigende Beispiele christlichen Handelns für Nachhaltigkeit sind mittlerweile an vielen Orten sichtbar und entsprechend dokumentiert, z. B. Klöster als Orte nachhaltigen Lebens (Gärtner, 2020), mit dem „Grünen Gockel“ ausgezeichnete kirchliche Einrichtungen oder zahlreiche sozial-ökologische Aktionen von kirchlichen Jugendverbänden (www.evangelische-jugend.de; www.bdkj.de; Birkel, 2002). Auch kann über Gruppierungen, wie z. B. Christians for Future, Kontakt zu christlichen Aktivist*innen hergestellt werden, die über ihre christlich motivierte Praxis berichten können, um hierüber Schüler*innen in einen "Zeugnis-Diskurs“ (Rappel, 2012, S. 225) einzubinden. In diesem Lernschritt geht es jedoch nicht nur um Ermutigung zum nachhaltigen Handeln, sondern auch um die kritische Reflexion der erlebten nachhaltigen Praxis anhand der zuvor im Unterricht erarbeiteten Stärken und Schwächen des Nachhaltigkeitsbegriffs.

Religiöse Bildung zielt somit darauf, dass Schüler*innen „,kritische Perspektiven zur Einschätzung von mächtigen Deutungen kennenlernen und entdecken, wie religiöse Deutungen an diesen Strukturen Anteil haben und zugleich etwas Eigenes in den Diskurs einspeisen." (Kumlehn, 2020, S. 430) (mit Bezug 
auf die Corona-Krise). Angesichts der immensen Herausforderungen der gegenwärtigen ökologischen Krise, sollen so die Schüler*innen ermutigt werden, bei Vergeblichkeitserfahrungen nicht zu resignieren. Vielmehr sollen sie befähigt werden, ihr Handeln als Teil einer refigurativen Praxis zu begreifen und sich für ein gutes Leben für alle in einer nachhaltigen Welt im Horizont der christlichen ReichGottes-Botschaft kritisch-emanzipativ einbringen zu können (Gärtner \& Herbst, 2020).

\section{Literaturverzeichnis}

Albert, Mathias; Hurrelmann, Klaus \& Quenzel, Gudrun (2019). Jugend 2019: Eine Generation meldet sich $z u$ Wort. Weinheim: Beltz.

Amlinger, Monika (2017). Gemeinschaft der Schöpfung auf dem Weg in die Zukunft Gottes. Jürgen Moltmanns Denken angesichts der neuen Enzyklika „Laudato si“ von Papst Franziskus. In KlausDieter Altmeppen, Frank Zschaler, Hans-Martin Zademach, Christoph Böttigheimer \& Markus Müller (Hg.), Nachhaltigkeit in Umwelt, Wirtschaft und Gesellschaft (S. 173-200). Wiesbaden: Springer.

Bederna, Katrin (2019). Every Day for Future. Theologische und religiöse Bildung für nachhaltige Entwicklung. Ostfildern: Matthias Grünewald.

Besand, Anja (2019). Hoffnung und Ihre Losigkeit. Politische Bildung im Zeitalter der Illusionskrise. In Anja Besand, Bernd Overwien \& Peter Zorn (Hg.), Politische Bildung mit Gefühl (S. 173-187). Frankfurt: Wochenschau Verlag.

Birkel, Simone (2002). Zukunft wagen - ökologisch handeln: Grundlagen und Leitbilder kirchlich-ökologischer Bildung im Kontext nachhaltiger Entwicklung. Münster: Lit.

Blühdorn, Ingolfur (2019). Die Gesellschaft der Nicht-Nachhaltigkeit. Skizze einer umweltsoziologischen Gegenwartsdiagnose. In Ingolfur Blühdorn, Felix Butzlaff, Michael Deflorian, Daniel Hausknost \& Mirijam Mock (Hg.), Nachhaltige Nicht-Nachhaltigkeit: Warum die ökologische Transformation der Gesellschaft nicht stattfindet (S. 65-142). Bielefeld: Transcript.

Blühdorn, Ingolfur; Butzlaff, Felix; Deflorian, Michael; Hausknost, Daniel \& Mock, Mirijam (Hg.) (2019). Nachhaltige Nicht-Nachhaltigkeit: Warum die ökologische Transformation der Gesellschaft nicht stattfindet. Bielefeld: Transcript. http://www.transcript-verlag.de/978-3-8376-4516-3

Böckenförde, Ernst-Wolfgang (1991). Die Entstehung des Staates als Vorgang der Säkularisation. In Ernst-Wolfgang Böckenförde (Hg.), Recht, Staat, Freiheit: Studien zur Rechtsphilosophie, Staatstheorie und Verfassungsgeschichte (S. 92-114). Frankfurt am Main: Suhrkamp.

Bork, Stefan \& Gärtner, Claudia (2016). Kirchengeschichtsdidaktik. Verortungen zwischen Religionspädagogik, Kirchengeschichte und Geschichtsdidaktik. Stuttgart: Kohlhammer.

Boschki, Reinhard (2008). Einführung in die Religionspädagogik. Darmstadt: Wissenschaftliche Buchgesellschaft.

Bröckling, Ulrich (2016). Das unternehmerische Selbst: Soziologie einer Subjektivierungsform (6. Auflage). Frankfurt: Suhrkamp.

Büttner, Gerhard (2012). Das „Reich Gottes“ im Klassenzimmer. Didaktische Erschließungen. Loccumer Pelikan, 22(1), 14-17.

Deeg, Janosch (10. Januar 2020). Plastik reduzieren - Klima schützen? Frankfurter Allgemeine Zeitung. https://www.faz.net/aktuell/wissen/erde-klima/plastik-reduzieren-klima-schuetzen-16575725.html

Deflorian, Michael (2019). Transformative Bewegungen? Nischenaktivismus zwischen Management und Überwindung der sozial-ökologischen Krise. In Ingolfur Blühdorn, Felix Butzlaff, Michael Deflorian, Daniel Hausknost \& Mirijam Mock (Hg.), Nachhaltige Nicht-Nachhaltigkeit: Warum die ökologische Transformation der Gesellschaft nicht stattfindet (S. 205-226). Bielefeld: Transcript.

Demirović, Alex (2013). Multiple Krise, autoritäre Demokratie und radikaldemokratische Erneuerung. PROKLA. Zeitschrift für kritische Sozialwissenschaft, 43(171), 193-215. https://doi.org/10.32387/prokla.v43i171.266 
Emprechtinger, Magdalena \& Papadopoulos, Helena (Hg.) (2020). Green Economy. Unterrichtsmaterialien zu Globaler Ökonomie. Wien: Kammer für Arbeiter und Angestellte.

Fuchs, Christian; Hommerich, Luisa; Nejechleba, Martin; Parnack, Charlotte; Siemes, Christof; Simon, Jana \& Sußebach, Henning. (2020). „Die versuchen, sich abzureagieren“. Rechtsextreme Netzwerke bei der Polizei. Die Zeit, 40. https://www.zeit.de/2020/40/rechtsextreme-netzwerke-polizei-chatsrassismus

Fuchs, Gotthard (1995). Gerichtsverlust. Katechetische Blätter, 120(3), 160-168.

Gäbel, Georg (2007). Mehr Hoffnung wagen (Vom Senfkorn) (Mk 4,30-32par). In Ruben Zimmermann (Hg.), Kompendium der Gleichnisse Jesu (S. 327-336). Gütersloh: Gütersloher Verlagshaus.

Gärtner, Claudia (2020). Klima, Corona und das Christentum. Religiöse Bildung für nachhaltige Entwicklung in einer verwundeten Welt. Bielefeld: Transcript.

Gärtner, Claudia \& Herbst, Jan-Hendrik (Hg.) (2020). Kritisch-emanzipatorische Religionspädagogik: Diskurse zwischen Theologie, Pädagogik und Politischer Bildung. Wiesbaden: Springer.

Gagel, Walter (1994). Untiefen der Katastrophendidaktik: Von der Ambivalenz des Begriffs „Schlüsselprobleme“. Politische Bildung. Beiträge zur wissenschaftlichen Grundlegung und zur Unterrichtspraxis, $27(2), 44-57$.

Gagel, Walter (2005). Geschichte der politischen Bildung in der Bundesrepublik Deutschland 1945-1989/90 (3. Auflage). Wiesbaden: VS Verlag für Sozialwissenschaften.

Göpel, Maja (2020). Unsere Welt neu denken: Eine Einladung. München: Ullstein Taschenbuch Verlag.

Hurrelmann, Klaus \& Albrecht, Erik (2020). Generation Greta: Was sie denkt, wie sie fühlt und warum das Klima erst der Anfang ist. Weinheim: Beltz.

Ideland, Malin (2019). The Eco-Certified Child: Citizenship and Education for Sustainability and Environment. Cham: Palgrave Pivot. https://doi.org/10.1007/978-3-030-00199-5

Ideland, Malin \& Malmberg, Claes (2015). Governing 'eco-certified children' through pastoral power: critical perspectives on education for sustainable development. Environmental Education Research, 21(2), 173-182. https://doi.org/10.1080/13504622.2013.879696

Kemfert, Claudia (2020). Mondays for Future: Freitag demonstrieren - am Wochenende diskutieren - ab Montag anpacken und umsetzen. Hamburg: Murmann.

Kluwick, Ursula \& Zemanek, Evi (Hg.) (2019). Nachhaltigkeit interdisziplinär: Konzepte, Diskurse, Praktiken: ein Kompendium. Wien: Böhlau Verlag.

Körtner, Ulrich (11. August 2020). Glaubenskrise. Der Gott der Klimaschützer. Frankfurter Allgemeine Zeitung. https://www.faz.net/aktuell/feuilleton/debatten/warum-die-akzeptanz-der-kirche-schwindet-16898466.html?premium

Kruse-Graumann, Lenelis (2003). Umweltverhalten - Handeln wider besseres Wissen? In Gotthilf Hempel \& Meinhard Schulz-Baldes (Hg.), Nachhaltigkeit und globaler Wandel: Guter Rat ist teuer (S. 175192). Frankfurt am Main: Lang.

Kumlehn, Martina (2020). Konfligierende Krisendeutungen und Krisennarrative: Deutungsmachtsensible Impulse religiöser Bildung in der Covid-19-Pandemie. Zeitschrift für Pädagogik und Theologie, 72(4), 427-441. https://doi.org/10.1515/zpt-2020-0049

Lachmann, Rainer (2016). Reich Gottes. In Rainer Lachmann, Gottfried Adam \& Werner H. Ritter (Hg.), Theologische Schlüsselbegriffe. Biblisch - systematisch - didaktisch, Theologie für Lehrerinnen und Lehrer 1 (6. Auflage) (S. 293-299). Göttingen: Vandenhoek \& Ruprecht.

Lindner, Konstantin (2007). In Kirchengeschichte verstrickt. Zur Bedeutung biographischer Zugänge für die Thematisierung kirchengeschichtlicher Inhalte im Religionsunterricht. Göttingen: Vandenhoek \& Ruprecht.

Mendl, Hans (2015). Modelle - Vorbilder - Leitfiguren. Lernen an außergewöhnlichen Biografien. Stuttgart: Kohlhammer.

Michelsen, Gerd; Grunenberg, Heiko \& Mader, Clemens (2015). Engagement durch Bildung für nachhaltige 
Entwicklung: Das Weltaktionsprogramm von Quantität zu Qualität. https://www.greenpeace.de/sites/www.greenpeace.de/files/publications/nachhaltigkeitsbarometer-bildung-20150522.pdf

Moltmann, Jürgen (1985). Gott in der Schöpfung: Ökologische Schöpfungslehre. Systematische Beiträge zur Theologie. München: Kaiser.

Müller, Peter; Büttner, Gerhard; Heiligenthal, Roman \& Thierfelder, Jörg (2002). Die Gleichnisse Jesu. Ein Studien- und Arbeitsbuch für den Unterricht. Stuttgart: Calwer.

Prescher, Thomas (2019). Das Nachhaltigkeitsdogma: Wie wir lassen, was wir tun sollten. Stuttgart: Kohlhammer.

Pufé, Iris (2017). Nachhaltigkeit (3. Auflage). Konstanz: UVK.

Rappel, Friederike (2012). Die Utopie des Gottesreiches als Chance für den Religionsunterricht. Berlin: Lit.

Raworth, Kate (2018). Die Donut-Ökonomie: Endlich ein Wirtschaftsmodell, das den Planeten nicht zerstört. München: Carl Hanser Verlag.

Reuter, Ingo (2020). „Fridays for Future“ - Über Klimaschwankungen im öffentlichen Diskurs angesichts des Widerstandes junger Menschen gegen den Totalitarismus der Sachzwänge. Zeitschrift für Pädagogik und Theologie, 72(3), 312-323. https://doi.org/10.1515/zpt-2020-0035

Sander, Hans-Joachim (2016). Raum nachhalten für menschliche Ohnmacht. Die Topologie der Zeichen der Zeit in Gaudium et spes. In Markus Patenge, Roman Beck \& Markus Luber (Hg.), Schöpfung bewahren: Theologie und Kirche als Impulsgeber für eine nachhaltige Entwicklung (S. 16-29). Regensburg: Friedrich Pustet.

Sobrino, Jon (1995). Die zentrale Stellung des Reiches Gottes in der Theologie der Befreiung. In Ignacio Ellacuría \& Jon Sobrino (Hg.), Mysterium liberationis: Grundbegriffe der Theologie der Befreiung (S. 461504). Luzern: Exodus.

Sobrino, Jon (2008). Christologie der Befreiung (2. Auflage). Ostfildern: Matthias Grünewald.

Theißen, Gerd \& Merz, Annette (2011). Der historische Jesus. Ein Lehrbuch (4. Auflage). Göttingen: Vandenhoeck \& Ruprecht.

UNESCO (2020). Framework for the implementation of Education for Sustainable Development (ESD) beyond. Paris. https://www.unesco.de/sites/default/files/2020-04/40\%20C\%2023\%20ESD.pdf

Vereinte Nationen (2015). Transformation unserer Welt. Die Agenda 2030 für nachhaltige Entwicklung. New York. https://www.un.org/depts/german/gv-70/band1/ar70001.pdf?OpenElement

Weiße, Wolfgang (2019). Reich Gottes. In Martin Rothgangel, Henrik Simojoki \& Ulrich H. J. Körtner (Hg.), Theologische Schlüsselbegriffe subjektorientiert - biblisch - systematisch - didaktisch. (Neuausgabe, S. 342-354). Göttingen: Vandenhoeck \& Ruprecht.

Yates, Luke (2015). Rethinking Prefiguration: Alternatives, Micropolitics and Goals in Social Movements. Social Movement Studies, 14(1), 1-21. https://doi.org/10.1080/14742837.2013.870883 